\title{
Humoral immune durability of IgG for anti-SARS-CoV-2 in the normal pregnant women in Wuhan, China
}

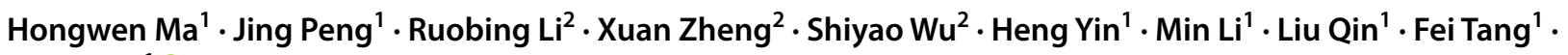 \\ Yun Zhao' ${ }^{1}$
}

Received: 11 May 2021 / Accepted: 10 July 2021 / Published online: 22 July 2021

(c) The Author(s), under exclusive licence to Springer Science+Business Media, LLC, part of Springer Nature 2021

\section{Dear Editors:}

Elucidating the duration of the $\mathrm{IgG} / \mathrm{IgM}$ antibodies against SARS-CoV-2 is important for clinical management and interpreting results from serological surveys [1]. Serological surveys have demonstrated that IgM antibody against SARS-CoV-2 is detected slightly earlier than IgG antibody and the $\operatorname{IgM} / \operatorname{IgG}$ exhibits excellent overall sensitivity and specificity [2]. The detection of IgG antibodies is more helpful for assessing the prognosis and recovery from symptomatic or asymptomatic infection and for evaluating the ability to resist reinfection. According to previous research, it is believed that pregnant women are more susceptible to virus infection due to alterations of immunity and anatomy [3]. How about the regression of IgG antibodies in the normal pregnant women after SARS-CoV-2 exposure? There is a lack of enough knowledge currently. We observed the epidemiology and longitudinal characterization of the positive IgG for SARS-CoV-2 in the normal pregnant women who have been treated for 9 months in our hospital, and the husbands of pregnant women and medical staff on the department of obstetrics were selected

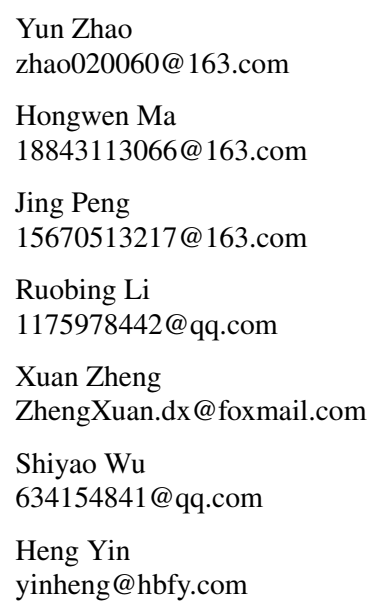

as the control. Our hospital is a tertiary level public hospital in Wuhan, Hubei Province, China. During the outbreak of COVID-19 infection, our hospital was a non-designated hospital including department for high fever to accept normal pregnant women and suspected pregnant patients.

From January 1, 2020, to March 31, 2020, a total of 6224 pregnant women gave birth in our hospital. Of the 6224 pregnant women, 70 cases were suspected pregnant women on admission according to pulmonary CT scan and routine blood test, who had symptoms of fever, cough, chest tightness, or gastrointestinal symptoms and were admitted to an isolated suite, and 19 cases were confirmed with COVID19 infection for being tested positive for SARS-CoV-2 via maternal throat swab test after admission, and then transferred to designated hospitals. During that time, the rate of COVID-19 infection in pregnant women in our hospital was $0.3 \%$ (19/6224). The diagnosis of COVID-19 was based on the seventh edition of the New Coronavirus Pneumonia Prevention and Control Protocol for the novel coronavirus disease 2019 (COVID-19) released by the National Health Commission of the People's Republic of China on March 4, 2020 [4].
Min Li

423611361@qq.com

Liu Qin

50729819@qq.com

Fei Tang

tangfei87169226@163.com

1 Department of ObstetricsMaternal, Child Health Hospital of Hubei Province, Tongji Medical College, Huazhong University of Science and Technology, No. 745, Wuluo Road, Hongshan District, Wuhan 430070, China

2 Department of Gynecology and Obstetrics, Wuhan University of Science and Technology, No. 2, Huangjiahu West Road, Hongshan District, Wuhan 430065, China 
From April 1 to December 30, 2020, all pregnant women and their accompanying members $(\leq 2$ per person, including husband) on admission in our hospital underwent throat swab test for SARS-CoV-2 and serological screening of antibodies IgM and IgG for SARS-CoV-2. If they did not complete those results, they would be admitted to an isolated suite. During that time, there were 15,953 pregnant women giving birth, and 15,671 accompanying husbands underwent throat swab test and serological screening of antibodies IgM and $\mathrm{IgG}$ (colloidal gold method) for SARS-CoV-2. All of them had negative polymerase chain reaction (PCR) testing and negative IgM for SARS-CoV-2. However, 106 pregnant women and 114 accompanying husbands had positive IgG for SARS-CoV-2, and the rates of positive IgG were $0.66 \%(106 / 15,953)$ and $0.73 \%(114 / 15671)$, respectively. Of the 106 pregnant women, only $5(4.72 \%)$ cases were early diagnosed with COVID-19 and received systematic isolation treatment; 18 (16.98\%) cases had fever, cough, chest tightness, or gastrointestinal symptoms. Of the 114 accompanying husbands having positive $\mathrm{IgG}, 7$ (6.14\%) cases were early diagnosed with COVID-19 and received isolation treatment, and 20 (17.54\%) cases had mild symptoms of fever, cough, chest tightness, or gastrointestinal symptoms.

From April 1 to April 30, 2020, all medical staff (385 members) in the department of obstetrics in our hospital underwent throat swab test and serological screening of $\mathrm{IgM}$ and IgG for SARS-CoV-2. None of the subjects was tested positive for nucleic acid and IgM, but 7 staff had positive IgG for SARS-CoV-2, with the rate of positive
IgG reaching $1.8 \%$ (7/385). Of those 7 staff with positive IgG, only 2 had mild fever and cough without any treatment 1 month ago, and no one was confirmed with COVID-19 infection. There was no difference in the positive rate of IgG in the normal pregnant women, in their accompanying husbands, and in the medical staff in the department of obstetrics $(Z=1.546, P=0.462$ by the chi-square test). The IgG-positive rate of pregnant women in April was significantly higher than that of confirmed cases from January to March $(Z=21.179, P=<0.001$ by the chi-square test).

Figure 1 shows the duration of positive $\operatorname{IgG}$ rate in the normal pregnant women and their accompanying husband. The change trend of the positive rate of $\operatorname{IgG}$ in the normal pregnant women was roughly similar to that of the general population (accompanying husbands) $(r=0.887$, $P=0.001^{<} 0.05$ by correlation analysis). The positive rate of IgG dropped slightly faster in April 1 to September 1 for both the normal pregnant women $\left(Z=41.852, P={ }^{<} 0.001\right.$ by the Cochran-Armitage Trend test) and their accompanying husbands $(Z=13.805, P=<0.001$ by the CochranArmitage Trend test), but in September 1 to December 31, it maintained at a low level for both the normal pregnant women $(Z=0.103, P=0.748>0.05$ by the Cochran-Armitage Trend test) and their accompanying husbands $(Z=1.651$, $P=0.199^{\prime} 0.05$ by the Cochran-Armitage Trend test).

It can be found from our study that in the early diagnosed cases of COVID-19, the positive rate of $\operatorname{IgG}$ for the normal pregnant women and their accompanying husbands was $4.72 \%$ and $6.14 \%$, and the rate of mild symptoms was
Fig. 1 The changes of IgG positive among pregnant women, their husbands, medical staff, and number of pregnant women

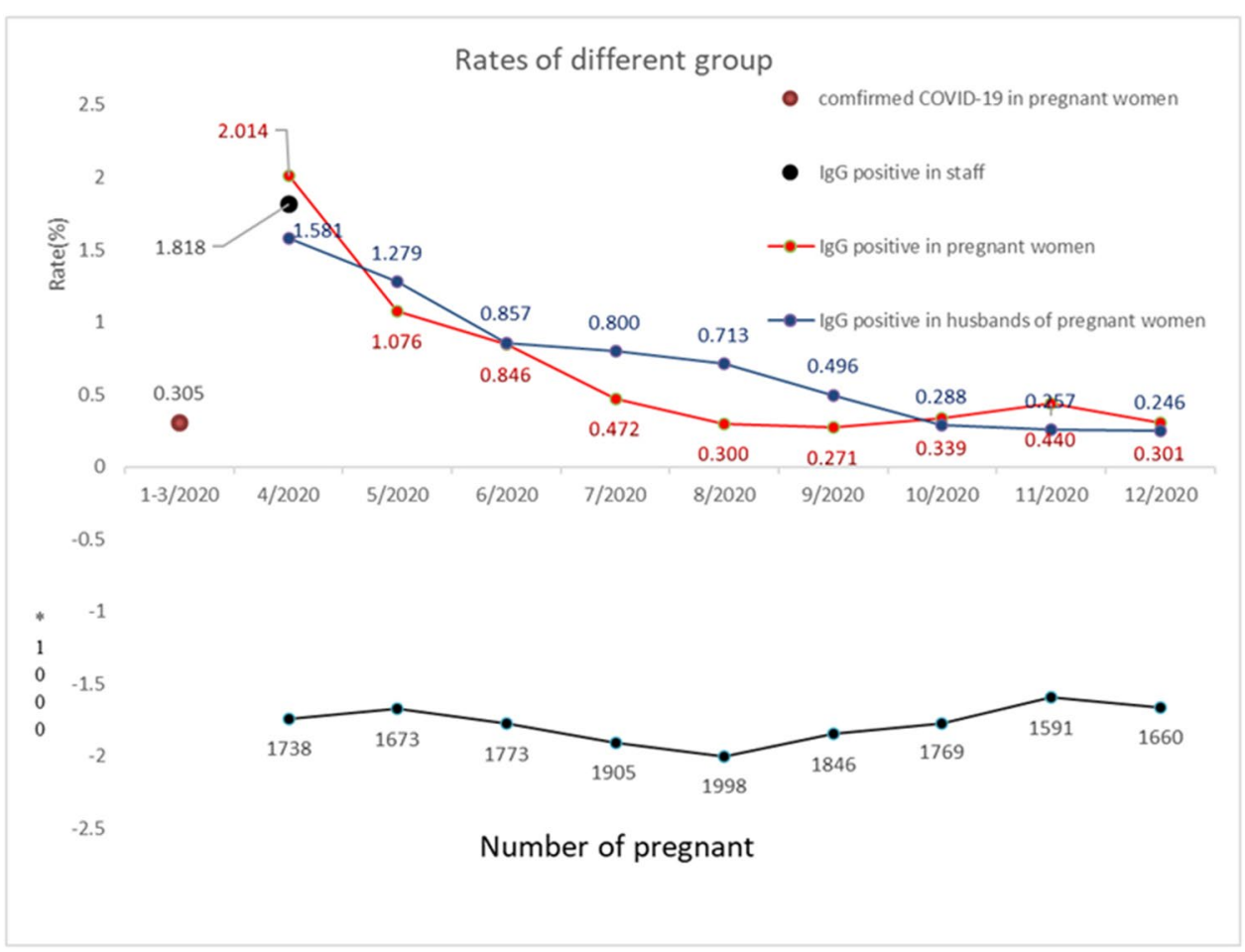


$16.98 \%$ and $17.54 \%$, respectively. He $\mathrm{Z}$ et al. found that $80 \%$ of people infected with SARS-CoV-2 were asymptomatic during the first wave of the pandemic, which is consistent with our results.

We can have the following discussion:

1. This trend of change is roughly consistent with the control of the domestic epidemic in China. Since April 2020, there has been no large-scale outbreak in China, especially in Wuhan where the number of new infection cases remained zero for long

2. The seroprevalence IgG antibody of SARS-CoV-2 varies at the population level from 3.9 to $5.6 \%$ at the beginning of universal test of antibodies $\mathrm{IgG} / \mathrm{IgM}$ and throat swab PCR test [5], but the positive rate of $\operatorname{IgG}$ was $1.581 \%$ in the normal pregnant women and $2.014 \%$ in their accompanying husbands. Such low positive rate may be due to the fact that our hospital was a non-designated hospital during the COVID-19 infection outbreak in Wuhan, and the pregnant women and their families were mostly in self-quarantine state during the lockdown period of Wuhan.

3. By March 2021, the full coverage of vaccine against SARS-COV-2 has been realized step by step. To date, no pregnant women have been advised to receive the COVID-19 vaccine for safety reasons. A study has shown that the immune status of pregnant women is completely different at different stages of pregnancy [6]. Therefore, if a pregnant woman wants to be vaccinated with new coronavirus antibodies, we should pay more attention to observe the safety.

This study was conducted based on only one birth center, rather than multiple centers, which is the major limitation of this study. In addition, there was no longitudinal follow-up for pregnant women whose $\operatorname{IgG}$ was positive. Finally, there was no antibody titer in the positive IgG pregnant women. All of above are worthy of further study.
Funding This work was supported by the Foundation from Hospital Association of Hubei Province (No: HBSG2020011) and Hubei Provincial Health and Family Planning Commission (No: WJ 2018H0139).

\section{Declarations}

Ethics approval The study design, the procedure of result communication, the information circular, and the questionnaire have been submitted to and approved by our hospital's ethics committee (ethical agreement number: [2020]IEC(XM002)).

Conflict of interest The authors declare no competing interests.

\section{References}

1. Maine GN, Lao KM, Krishnan SM, Afolayan-Oloye O, Fatemi S, Kumar S, VanHorn L, et al. Longitudinal characterization of the IgM and $\mathrm{IgG}$ humoral response in symptomatic COVID-19 patients using the Abbott Architect. J Clin Virol. 2020;133:104663.

2. Lumley SF, Eyre DW, McNaughton AL, Howarth A, Hoosdally S, Hatch SB, Kavanagh J, et al. SARS-CoV-2 antibody prevalence, titres and neutralising activity in an antenatal cohort, United Kingdom, 14 April to 15 June 2020. Euro Surveill. 2020;25(24):2001721.

3. Schwartz DA, Graham AL. Potential maternal and infant outcomes from (Wuhan) coronavirus 2019-nCoV infecting pregnant women: lessons from SARS, MERS, and other human coronavirus infections. Viruses. 2020;12(2):194.

4. China NHC. New Coronavirus Pneumonia Prevention and Control Protocol. 7th ed. National Health Commission of the People's Republic of China; 2020. Accessed 4 March 2020.

5. He Z, Ren L, Yang J, Guo L, Feng L, Ma C, Wang X, et al. Seroprevalence and humoral immune durability of anti-SARS-CoV-2 antibodies in Wuhan, China: a longitudinal, population-level, cross-sectional study. Lancet. 2021;397:1075-84.

6. Vojtek I, Dieussaert I, Doherty TM, Franck V, Hanssens L, Miller J, Bekkat-Berkani R, et al. Maternal immunization: where are we now and how to move forward? Ann Med. 2018;50:193-208.

Publisher's note Springer Nature remains neutral with regard to jurisdictional claims in published maps and institutional affiliations. 